\section{EDUCAÇÃO}

V.7 $\cdot$ N.3 • Abril/Maio/Junho - 2019

ISSN Digital: 2316-3828

ISSN Impresso: 2316-333X

DOI: 10.17564/2316-3828.2019v7n3p55-66
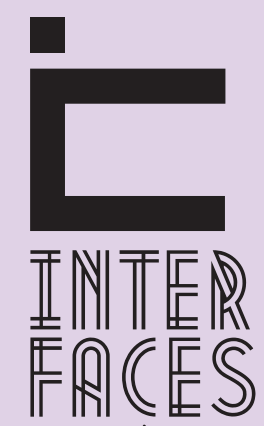

CIENTÍFICAS

\title{
TIPOS DE QUESTÕES PARA TESTES DE PROFICIEENCIA DAS HABILIDADES DE LEITURA E ESCRITA DE LÍNGUA INGLESA
}

TYPES OF QUESTIONS FOR PROFICIENCY TESTS OF BOTH READING AND WRITING SKILLS IN THE ENGLISHLANGUAGE

TIPOS DE PREGUNTAS PARA PRUEBAS DE COMPETENCIA DE LAS HABBLLDADES DE LECTURA Y ESCRITURA ENEL IDIOMA INGLÉS

Diego Martins Pinto Guedes ${ }^{1}$ Fabiola Coneglian ${ }^{2}$

\section{RESUMO}

O objetivo deste estudo se deu no aprofundamento dos conceitos teóricos e práticos sobre avaliações de proficiência, com vistas a identificar o que os testes de proficiência consideram como relevante na avaliação das habilidades comunicativas, no contexto de língua inglesa. O delineamento metodológico foi de cunho bibliográfico constituído em estudo do tipo estado da arte (PICHETH, 2007; ROMANOWSKI, 2006), também referido como revisão narrativa (ELIAS et al., 2012). Ao longo do trabalho, os variados conceitos de avaliação e características das habilidades comunicativas foram ponderados. Dentre os testes de proficiências - TOEFL (2015), TOEIC (2015), IELTS (2015), FCE (2015), CAE (2015) e CPE (2015) - foram investigados e identificados neles quais os tipos de questões mais recorrentes, destacando-se 15 tipos, os quais foram explanados no estudo.

\section{PALAVRAS-CHAVE}

Avaliação. Testes de Proficiência. Tipos de Questões. 


\section{ABSTRACT}

The aim of this study was to deepen the theoretical and practical concepts of proficiency tests in order to identify what they consider as relevant in the evaluation of communicative skills in the context of English language. The methodological design was based on bibliographic nature consisting in studies of state of the art (PICHETH, 2007; ROMANOWSKI, 2006), also referred to as narrative review (ELIAS et al, 2012). Throughout the paper, different concepts concerning the tests as well as features of communicative skills were discussed. Proficiency tests - TOEFL (2015), TOEIC (2015), IELTS (2015), FCE (2015), CAE (2015) e CPE (2015) - were investigated in order to identify the most frequent question types; out of them, 15 types were identified and detailed in the study.

\section{KEYWORDS}

Tests. Proficiency tests. Question types.

\section{RESUMEN}

El objetivo de este estudio fue profundizar los conceptos teóricos y prácticos de las pruebas de competencia para identificar lo que consideran relevante en la evaluación de las habilidades comunicativas en el contexto de la lengua inglesa. El diseño metodológico se basó en la naturaleza bibliográfica consistente en estudios de estado del arte (PICHETH, 2007; ROMANOWSKI, 2006), también conocido como revisión narrativa (ELIAS et al, 2012). A lo largo del artículo, se discutieron diferentes conceptos relacionados con las pruebas, así como características de las habilidades comunicativas. Pruebas de competencia - TOEFL (2015), TOEIC (2015), IELTS (2015), FCE (2015), CAE (2015) e CPE (2015) - fueron investigadas con el fin de identificar los tipos de preguntas más frecuentes; entre ellos, 15 tipos fueron identificados y detallados en el estudio.

\section{PALABRAS CLAVE}

Pruebas. Pruebas de competencia. Tipos de preguntas. 


\section{INTRODUÇ̧̃̃o}

Este estudo diz respeito a um aprofundamento dos conceitos teóricos e práticos sobre avaliações de proficiência, de modo a termos acesso a modelos de questões para elaboração de avaliações, com o objetivo de saber o que os testes de proficiência consideram como relevante na avaliação das habilidades comunicativas.

Esta é uma pesquisa de cunho bibliográfico constituído em estudo do tipo estado da arte (PICHETH, 2007; ROMANOWSKI, 2006), também referida como revisão narrativa (ELIAS et al., 2012), uma vez que estabelece relação com produções anteriores, identifica temática recorrente, aponta novas perspectivas, consolida uma área de conhecimento, constitui-se orientações de práticas pedagógicas, bem como define parâmetros para uma determinada área.

A seguir, faremos uma conceituação sobre as avaliações e suas vertentes. Discutiremos sobre proficiência linguística e as quatro habilidades comunicativas no ensino de línguas. Apresentaremos as avaliações de proficiência linguística amplamente conhecidas e exploraremos as características e objetivos de cada habilidade comunicativa avaliada. Explicitaremos os tipos de questões mais utilizados em testes de proficiência.

\section{CONCEITUANDO A AVALIAÇÃO}

Quando definidas no âmbito educacional, as avaliações são usadas para medir o quanto do material, atribuído aos alunos, está sendo apreendido e o quão bem os discentes estão alcançando suas respectivas metas e objetivos almejados.

A fim de compreender as avaliações de modo mais amplo, fora necessário identificar e conceituar suas vertentes, em inglês, evaluation, assessmente test, que apesar de suas traduções para língua portuguesa serem avaliação, trazem consigo diferentes aplicações e conotações, como veremos a seguir:

(1) evaluation diz respeito ao processo de fazer julgamentos com base em critérios e evidências.

Segundo o dicionário virtual Oxford Dictionaries, o termo faz referência à realização de um determinando julgamento acerca de quantidade, número ou valor de algo.

(2) assessment diz respeito ao processo de documentar conhecimento, habilidades, atitudes e crenças, geralmente em termos mensuráveis. 0 objetivo central está em realizar melhorias, ao invés de simplesmente julgar. Neste processo, busca-se descrever, coletar, gravar, pontuar e interpretar informações que permeiam a aprendizagem.

(3) test, exam ou quiz diz respeito ao processo de avaliar o conhecimento de alguém sobre algo e determinar o quê um indivíduo conhece ou aprendeu, em uma tentativa de identificar o nível de tal habilidade e/ou conhecimento. Segundo o dicionário virtual Oxford Dictionaries, testing faz referência ao procedimento destinado a determinar a qualidade, desempenho e/ou confiabilidade de algo, como, por exemplo, um exame escrito ou falado de proficiência linguística de uma pessoa - caso este de 
nosso estudo. Cabe ressaltar que o termo test se destaca por seu tom mais generalista, enquanto exam se destaca por maior formalidade e quiz, por sua vez, pela brevidade e informalidade.

McNamara (2000) afirma que ao longo da história as pessoas têm sido testadas para provar suas capacidades ou para estabelecer suas credenciais. E como exemplo teríamos os diversos exames de proficiência linguística, uma vez que buscam verificar como os candidatos se desempenham em relação a um nível minimamente desejado dentro de padrões estabelecidos, participando, assim, de seleções que acabam por controlar o acesso a muitos papéis socialmente importantes.

\section{PROFICIÊNCIA LINGUISTIICA}

Ao articular sobre proficiência linguística, corroboramos com Scaramucci (2000), que distingue a proficiência em duas modalidades: a do uso técnico e a do uso não técnico. A acepção do uso não técnico diz respeito a ponderações de teor impressionista, baseados em visões mais holísticas da linguagem, e leva à interpretação de proficiência como um conceito estável e singular. Comumente, o conceito de proficiência, visto sob essa visão, é baseado em descritores mais gerais e representa uma separação entre indivíduos proficientes e não proficientes.

Por outro lado, na acepção do uso técnico, o termo proficiência é utilizado no âmbito de controle operacional da língua, variando conforme a especificidade da situação de uso da mesma, englobando uma gradação de níveis, ou seja, oscilando do menos proficiente ao mais proficiente. Assim, segundo Scaramucci (2000), em vez de uma proficiência única, absoluta, monolítica, baseada naquela do falante ideal, teríamos várias, dependendo da situação de uso da língua.

Inteiramos, com base em Stern (1987), que a proficiência pode ser apresentada como resultado de um conceito multifacetado, isto é, que abrange o conhecimento linguístico e sociolinguístico, bem como a habilidade ou capacidade de colocar esse conhecimento em uso, por meio das habilidades comunicativas.

\subsection{HABILIDADES COMUNICATIVAS}

Consideram-se por habilidades comunicativas, as distintas capacidades que se tem para o estabelecimento da comunicação. Cabe aqui ressaltar que a comunicação diz respeito a transmissão de uma determinada mensagem, num dado conjunto de formas semanticamente significativas, por meios pelos quais o remetente da determinada mensagem se faz entendido pelo destinatário, a partir de uma produção comunicativa. Assim como as formas de comunicação, outro fator decisivo que necessita existir é a compreensão, pois não basta apenas haver interação entre remetente e destinatário numa dada mensagem, se a comunicação só passa a existir quando há real entendimento denotativo entre as duas partes. A seguir, explicitam-se as quatro habilidades comunicativas: a de compreensão oral (listening), a de produção oral (speaking), a de compreensão escrita (leading) e a de produção escrita (writing). 


\subsubsection{LISTENING}

O listening, em português compreensão auditiva, refere-se ao ato de ouvir. No entanto, tal ato representa mais que somente uma habilidade física, significa ouvir com atenção e, assim, entender o objetivo daquilo que se está ouvindo, isto é, ouvir com um propósito. Segundo Pauk e Owens (2013, p. 170), um bom listening é construído em três pilares conhecidos como Triple-A Listening: atitude (attitude), atenção (attention) e ajuste (adjustment).

Esta habilidade diz respeito à absorção das palavras e sons que chegam direto ao cérebro, direcionando-nos a entender os fatos e as ideias que estão sendo reproduzidas. Para o bom desempenho dessa habilidade, muita atenção e foco são demandados. Requerer concentração, traduz-se na focalização de seus pensamentos além de um problema particular. A pessoa que incorpora o listening com essa intensidade obtêm uma habilidade ativa.

\subsubsection{SPEAKING}

O speaking, em português produção de fala, é um processo interativo de construção de significados que envolve as ações de receber e processar informações. A interação acontece, dependendo dos propósitos dos indivíduos, bem como dos elementos da língua que lhes convêm, como, por exemplo, suas experiências, personalidades e ambientes socioculturais. Geralmente, ocorre de modo espontâneo e envolvente. No entanto, o discurso não é sempre imprevisível, pois o indivíduo pode prepará-lo antes de executá-lo.

As funções de linguagem tendem a direcionar para certas situações, como pedir um favor, ou passar instruções. Há uma grande diversificação nos mais variados níveis de fala. Cada indivíduo, além de conhecer aquilo que fala, conhece, também, aquilo que os outros falam, motivo este pelo qual os indivíduos são capazes de dialogar com pessoas dos mais variados graus de cultura, embora nem sempre a linguagem entre os mesmos seja exatamente igual.

Essa habilidade de produção oral, não requer somente saber pontos gramaticais, pronúncia e vocabulário, também se faz necessário saber quando, o porquê e quais são as maneiras de utilizar. Isto porque os discursos têm suas próprias características, estruturas e convenções, diferentemente da linguagem escrita. Sintetizar essas habilidades e conhecimentos resulta na obtenção do sucesso na produção oral ativa. Além disso, um falante deve ser capaz de antecipar e assim produzir um discurso esperado, escolhendo vocabulário e estruturas corretas para descrever determinadas coisas ou situações em um determinado contexto.

\subsubsection{READING}

O Reading, em português compreensão de leitura, é um processo cognitivo de decodificação dos símbolos para construção de um significado. Quando lemos, usamos nossos olhos para receber os símbolos (letras, pontuações e espaços) e usamos o cérebro para convertê-los em palavras, frases e parágrafos que correspondam a algo semanticamente inteligível. Pode ocorrer em silêncio (em nossa mente) ou em voz alta (para outrem). 
Esta é uma habilidade receptiva, uma vez que por ela recebemos informações. Mas no processo de leitura, recorremos a habilidade da fala para que possamos pronunciar as palavras.

Este processo é considerado multifacetado, posto que nele incluem-se identificação das palavras, compreensão, fluência e motivação. Diz respeito à aquisição da língua, da comunicação e da divisão de ideias e informações. A interação do leitor com o texto e moldada de acordo com seu conhecimento social e cultural, mas também requer prática, desenvolvimento, refinamento e análises críticas. Não há regras concretas quanto ao reading, tido como um processo complexo, não devemos restringir a somente uma ou duas conceituações. Leitores precisam ter uma variedade de estratégias para decodificação (tradução dos símbolos) e compreensão. Os mesmos podem usar o contexto para identificar palavras desconhecidas com vistas a interagir com outras palavras que já conhecem. A leitura pode ser feita a partir de variados gêneros, desde símbolos musicais e imagens, até mesmo sinais táteis.

\subsubsection{WRITING}

O writing, em português produção escrita, é um processo de empregar os símbolos (letras, pontuações e espaços) para conectar pensamentos e ideias de forma legível. Alguns estudos consideram a escrita como um complemento para o discurso e a fala. Para escrever claramente é essencial entender o sistema básico da linguagem. Na língua inglesa, isso inclui conhecimentos em gramática, pontuação, bem como de estruturas frasais e textuais. Vocabulário é também necessário, assim como ortografia e aspectos gráficos.

Dentro de um sistema de linguagem, a escrita se baseia em muitas das mesmas estruturas do discurso falado, como vocabulário, gramática e semântica, com a dependência adicional de um sistema de signos ou símbolos.

\section{TIPOS DE QUESTÕES EM TESTES PROFICIÊNCIA}

Nesta sessão, pretendemos investigar, identificar e explicitar quais os tipos de questões são mais recorrentes nos testes de proficiência: TOEFL (2015), TOEIC (2015), IELTS (2015), FCE (2015), CAE (2015) e CPE (2015), no que tange às habilidades de reading (compreensão leitora) e writing (produção escrita). Com este propósito, abordaremos em que consiste tais questões e possíveis aplicações no contexto da avaliação de proficiência de língua inglesa.

\subsection{TEXTO COM LACUNAS}

Uma questão do tipo open cloze (em português, texto com lacunas) consiste em um texto em língua estrangeira com algumas lacunas (em inglês, blanks ou cloze), cada uma das quais representa uma palavra ausente. Sendo assim, o respondente deve escrever a palavra correta para cada lacuna existente. Este modelo é geralmente utilizado para avaliar vocabulário e gramática da língua estrangeira. 


\subsection{VERDADEIRO OU FALSO}

Uma questão do tipo true or false (em português, verdadeiro ou falso) consiste em um número de declarações referentes a um texto ou contexto fornecido. Sendo assim, o respondente deve assinalar uma das seguintes opções: verdadeiro (se a declaração condiz) ou falso (se a declaração não condiz), levando em consideração somente as informações fornecidas. Alguns testes de proficiência trazem ainda uma terceira opção: inexistente (se a declaração não condiz nem contradiz, simplesmente a declaração não é citada ou referida), com vistas a aumentar a exigência de criticidade por parte do respondente, que deve ter muito cuidado para não usar qualquer informação extra, que julgue conhecer sobre o tópico, ao escolher sua resposta. Este modelo é geralmente utilizado para testar a capacidade do respondente de reconhecer informações específicas fornecidas em um determinado texto ou contexto linguístico-cultural.

\subsection{MÚLTIPLA ESCOLHA}

Uma questão do tipo multiple-choice (em português, múltipla escolha) consiste em um texto e/ou uma pergunta seguida de quatro respostas alternativas (A, B, C ou D). Sendo assim, dadas as informações, o respondente deve escolher a opção considerada correta. Para aumentar o grau de dificuldade há possibilidade do aceite de uma, duas, três e/ou quatro opções corretas; contudo, nos testes analisados apenas uma opção é considerada correta. Este modelo é geralmente utilizado para testar a capacidade de leitura minuciosa, opinião, tom, propósito, ideia principal e/ou implicação linguística.

\subsection{MÚLTIPLA ESCOLHA COM LACUNAS}

Uma questão do tipo multiple-choice cloze (em português, múltipla escolha com lacunas) consiste em um texto com algumas lacunas numeradas, cada uma das quais representa uma palavra ou frase ausente. $O$ texto é seguido de quatro possíveis respostas para cada lacuna. Sendo assim, o respondente deve escolher a resposta correta ( $, B, C$ ou D), isto é, a alternativa que melhor completa o sentido que fora omitido pela lacuna. Este modelo é geralmente utilizado para testar o conhecimento vocabular do respondente, mais especificamente idioms (expressões idiomáticas), collocations (colocações lexicais), shades of meaning (diferenças sutis em uso lexical), phrasal verbs (colocação preposicional ou adverbial alterando o significado do verbo), fixed phrases (expressões fixas) etc.

\subsection{RESPOSTA DISCURSIVA}

Uma questão do tipo writing short answer (em português, resposta curta discursiva), consiste em um questionamento aberto com base em um texto, tema ou contexto fornecido. Sendo assim, o respondente deve responder de modo a dissertar objetivamente. Este modelo é geralmente utilizado para avaliar a capacidade do respondente em identificar detalhes factuais no texto ou expressar suas considerações acerca de um determinado tópico. 


\subsection{ASSOCIAÇÃO}

Uma questão do tipo matching (em português, combinação ou associação) consiste em duas colunas, uma contendo breves descrições e outra contendo palavras. Sendo assim, o respondente deve associar as colunas, criando pares de palavras com suas respectivas descrições. São inúmeras as variações deste tipo de questão, onde as colunas podem conter fotos, ilustrações, palavras, frases, áudios, entre outros itens passíveis de combinação. Este modelo é geralmente utilizado para avaliar vocabulário, gramática e compreensão.

\subsection{FORMAÇÃO LEXICAL}

Uma questão do tipo word formation (em português, formação lexical) consiste em um texto contendo lacunas, que representam palavras ausentes. Após a lacuna ou ao final da sentença há uma keyword (palavra-chave) entre parênteses que deve ser empregada, porém em distinta forma. Sendo assim, o respondente deve compreender a frase e modificar a keyword de alguma forma que complete a frase corretamente. Este modelo é geralmente utilizado para avaliar conhecimento de vocabulário e gramática, mais especificamente de classes gramaticais.

\subsection{MODIFICAÇÃO FRASAL}

Uma questão do tipo keyword transformation (em português, modificação frasal por palavra-chave) consiste em uma frase seguida por uma keyword (palavra-chave) e uma segunda frase com uma lacuna. Sendo assim, o respondente deve compreender minuciosamente a primeira frase e usar a keyword para completar a segunda frase, usando de três a seis palavras, de modo que a segunda frase mantenha o mesmo significado da primeira frase. Este modelo é geralmente utilizado para avaliar vocabulário, gramática e composição escrita coerente e coesa.

\subsection{MÚLTIPLA ASSOCIAÇ̃̃O TEXTUAL}

Uma questão do tipo cross-text multiple matching (em português, múltipla associação textual) consiste em textos curtos com alternativas de múltipla combinação. Sendo assim, o respondente deve ler todos os excertos textuais fornecidos com vistas a combinar imagens, frases ou elementos com os excertos; às vezes, até mesmo combinar os excertos entre si. Este modelo é geralmente utilizado para mensurar o nível de compreensão e testar a capacidade de comparar opiniões e atitudes ao longo dos textos.

\subsection{TEXTO DESORDENADO COM LACUNAS}

Uma questão do tipo cluttered gapped-text (em português, texto desordenado com lacunas) consiste em uma página de texto com algumas lacunas numeradas que representam parágrafos ausen- 
tes. Após o texto, há alguns excertos numerados, porém desordenados. Sendo assim, o respondente deve ler o texto com lacunas e os excertos numerados com vistas a decidir qual excerto melhor se adapta a cada lacuna. Este modelo é geralmente utilizado para avaliar a capacidade de compreensão da estrutura textual e do desenvolvimento de textos de diferentes gêneros, além de exigir do respondente um determinando nível de produção escrita.

\subsection{MÚLTIPLA ASSOCIAÇ̃̃O}

Uma questão do tipo multiple matching (em português, múltipla associação) consiste em questionamentos, com múltiplas alternativas, seguidos de um texto único ou vários textos curtos. Assim, o respondente deve combinar as alternativas com os elementos do texto ou dos textos. Este modelo é geralmente utilizado para testar a leitura crítica com intuito de obter informações específicas, detalhes e opiniões.

\subsection{PREENCHIMENTO FRASAL}

Uma questão do tipo sentence completion (em português, preenchimento frasal) consiste em frases incompletas. Sendo assim, o respondente deve preencher todas as frases com as informações ausentes por meio do áudio que será executado. Este modelo é geralmente utilizado para testar compreensão oral do respondente e sua capacidade de identificar detalhes relevantes.

\subsection{REDIGIR O FINAL DA FRASE}

Uma questão do tipo writing sentence endings (em português, redigir o final da frase) consiste em frases com o início já provido, porém sem final definido. Sendo assim, o respondente deve compreender profundamente a frase parcialmente fornecida e inventar um final para a mesma. Este modelo é geralmente utilizado para testar a capacidade de compreender as ideias principais e a capacidade de completar ideias de forma coesa, coerente e criativa.

\section{14 CORRETO OU INCORRETO}

Uma questão do tipo correct or incorrect (em português, correto ou incorreto) consiste em um número de declarações referentes a um texto ou contexto fornecido. Sendo assim, o respondente deve analisar e assinalar uma das seguintes opções: correto (se a declaração está completamente certa) ou incorreto (se a declaração está parcialmente certa, isto é, possui erros), levando em consideração somente as informações fornecidas. Este modelo é geralmente utilizado para testar a capacidade do respondente de reconhecer informações específicas fornecidas em um determinado texto, mas principalmente avaliar se o respondente possui elevado conhecimento sobre um determinado tópico gramatical. 


\subsection{ESCRITA DE PERGUNTAS}

Uma questão do tipo writing questions (em português, escrita de perguntas) consiste no fornecimento de respostas prontas com espaço para elaboração do questionamento. Sendo assim, o respondente deve ler com muita atenção a resposta dada para que assim possa escrever uma pergunta de alta precisão. Este modelo é geralmente utilizado para testar o nível de compreensão e a capacidade de objetividade na indagação durante a comunicação.

\section{CONSIDERAÇ̃̃ES FINAIS}

Por meio deste trabalho, pôde-se estudar profundamente os conceitos que premeiam o âmbito da avaliação linguística, bem como conhecer detalhadamente os testes reconhecidos internacionalmente. Além disso, esta pesquisa mostra-se relevante e um excelente referencial a professores quando na elaboração de suas avaliações linguísticas.

\section{REFERÊNCIAS}

CAE - Certificate in Advanced English. Disponível em: http://www.cambridgeenglish.org/exams/ advanced/exam-format. Acesso em: 8 nov. 2015.

CPE - Certificate of Proficiency in English. Disponível em: http://www.cambridgeenglish.org/ exams/proficiency/ exam-format. Acesso em: 29 nov. 2015

ELIAS, C. S. et al. Quando chega o fim? Uma revisão narrativa sobre terminalidade do período escolar para alunos deficientes mentais. Revista Electrónica en Salud Mental, SMAD: Alcohol y Drogas, v. 8, n. 1, p. 48-53, 2012.

FCE - First Certificate in English. Disponível em: http://www.cambridgeenglish.org/exams/first/ exam-format. Acesso em: 18 nov. 2015

IELTS - International English Language Testing System. Disponível em: http://takeielts. britishcouncil.org/prepare-test/understand-test-format. Acesso em: 03 de nov. de 2015

MCNAMARA, T. Language testing. Oxford: OUP, 2000.

OXFORD Dictionaries. Disponível em: http://www.oxforddictionaries.com. Acesso em: 20 dez. 2015. 
PAUK, W.; OWENS, R. J. Q. How to Study in College. Wadsworth Publishing; 11 edition, 9 January 2013.

PICHETH, F. M. PeArte: um ambiente colaborativo para a formação do pesquisador que atua no ensino superior por meio da participação em pesquisas do tipo estado da arte. 2007. 139f. Dissertação (Mestrado em Educação) - Pontifícia Universidade Católica do Paraná, Curitiba, 2007. Disponível em: http://www.biblioteca.pucpr.br/tede/tde_busca/arquivo.php?codArquivo=828. Acesso em: 10 nov. 2015.

ROMANOWSKI, J. P.; ENS, R. T. As pesquisas denominadas do tipo "estado da arte" em educação. Diálogo Educacional, v. 6, n. 19, p. 37-50, set./dez., 2006.

SCARAMUCCI, M. V. R. Avaliação: mecanismo propulsor de mudanças no ensino/aprendizagem de língua estrangeira. Contexturas, n. 4, p. 115-124, 1999/2000.

SCARAMUCCI, M. V. R. Proficiência em LE: considerações terminológicas e conceituais. Trabalhos de Lingüística Aplicada 36, p. 11-22, 2000.

STERN, H. H. Fundamental concepts in language teaching. Oxford: Oxford University Press, 1987.

TOEFL. Test of english as a foreign language. Disponível em: http://www.ets.org/toefl/ibt/about/ content. Acesso em: 16 nov. 2015.

TOEIC. Test of english for international communication. Disponível em: https://www.ets.org/toeic/ speaking_writing/ about/content. Acesso em: 21 nov. 2015. 
1 Doutorando em Educação pela UNR/Argentina; Mestre em Processos e Distúrbios da Comunicação pela FOB/ USP; Especialista em Ensino de Língua Estrangeiras com foco em Inglês pela USC e em MBA Marketing pela FGV; Bacharel em Tradução (Português/Inglês) pela USC; Tradutor profissional e Professor de Inglês; Diretor da instituição de ensino Master Method; Escritor da série Master Method, livros didáticos de língua inglesa; Possui grande interesse de pesquisa nas áreas de ensino e aprendizagem de línguas, fonoaudiologia, tradução e linguística, bem como tópicos relacionados à comunicação e administração em geral. E-mail: dimaguedes@gmail.com

2 Especializando-se em Psicopedagogia pela USC; Licenciatura em Letras (Português/Inglês) pelo instituto IMES. E-mail: coneglian086@hotmail.com

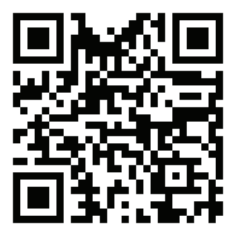

A autenticidade desse artigo pode ser conferida no site https://periodicos. set.edu.br

\section{Como citar este artigo:}

GUEDES, Diego Martins Pinto; CONEGLIAN, Fabiola. Tipos de questões para testes de proficiência das habilidades de leitura e escrita de língua inglesa. Interfaces Científicas Educação, Aracaju, v. 7, n. 3, p.55-66, abr. 2019. DOI: 10.17564 /2316-3828.2018v7n1p55-66 Acesso em: 24 mar. 2019.

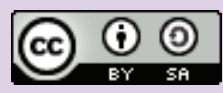

Este artigo é licenciado na modalidade acesso abertosob a Atribuição-Compartilhalgual CC BY-SA

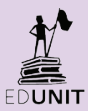

